\title{
A Duty to Recontact in the Context of Genetics: Futuristic or Realistic?
}

\author{
Corrette Ploem, ${ }^{a}$ Colin Mitchell, ${ }^{b}$ Wim van Harten ${ }^{c}$ and Sjef Gevers ${ }^{d}$ \\ a Academic Medical Center, Amsterdam, The Netherlands \\ b Doctoral student, Health Law, Amsterdam University Medical Center, \\ Amsterdam, The Netherlands \\ c Professor, Quality Management and Governance, University of Twente, \\ Twente, The Netherlands; Dept. Psychosocial Research and Epidemiology, \\ The Netherlands Cancer Institute, Amsterdam, The Netherlands \\ d Emeritus Professor of Health Law, University of Amsterdam, Amsterdam, \\ The Netherlands
}

\begin{abstract}
Medical genetic testing, 'next generation sequencing', is increasingly generating data that could become useful for patients after they have been discharged from care. If new information is discovered that links a disease to a specific mutation, do health professionals have a legal duty to recontact their patients? Apart from other concerns (such as respecting the patient's right not to know), in many cases, this would require re-evaluation or re-analysis of the data. Taking such issues into account, we conclude that, at least at this point in time, it is not arguable that there is an unconditional duty of this kind. Health professionals should always do what can be reasonably expected from them to do justice to the patient's right to information. When there is reason to believe that recontacting would be of significant clinical relevance for the patient, they should do so, unless efforts and costs involved would be disproportional.
\end{abstract}

\section{Keywords}

genetic testing - duty to recontact - patient's right to information - updating previous test results 
There have always been circumstances in which healthcare providers have felt obliged to renew contact with patients because new information has become available concerning their health. It is possible that such recontacting is relevant for current patients who have a long-term relationship with their physician, but do not have contact with him or her on a regular basis. However, it can be particularly important for former patients (or their relatives) who have been previously discharged from care and are no longer in an ongoing relationship with their healthcare provider.

Recontacting may serve different purposes. Examples in the past include the need to offer testing and care to individuals after discovering a risk of transmitting infection during care, medical device warnings and recall, new knowledge about adverse reactions in drugs, or, the simple fact that a new test or treatment had become available that might be important for a patient or a certain group of patients. Some of these cases, in particular when warning patients would protect them for foreseeable danger or to avoid potential harm (e.g., in case of failing medical devices or dangerous drugs), became lawsuits. In most cases, physicians were not considered to have a legal obligation to inform former patients about new developments related to their health problems, taking into account the effort required to recontact patients already discharged from care.

Now, the issue of recontacting is back on the agenda in a relatively new form, raising questions that have not been sufficiently addressed in the past. This is particularly related to developments in genetics and genomics, especially 'next generation sequencing' (NGS) and 'whole genome sequencing' (WGS), technologies currently being introduced in clinical practice. This results in a growing number of patients having parts of - or their whole - exome (WES) or genome sequenced in different settings (before conception, pre-natal, screening). Whereas an individual's DNA essentially will remain the same (unless there is tumour development), the knowledge about the medical significance of the collected data continues to evolve over time: what has been interpreted

1 The research for this article was carried out as part of the TANGO-project (Technology Assessment of Next Generation Sequencing in personalised oncology), financed by ZonMw, The Hague, The Netherlands. Although the TANGO-project is specifically focused on NGS and wGs-based clinical research in the field of lung cancer and of melanoma, this article addresses the topic of recontacting in a more general way. 
as innocent or not significant today, may be reclassified as significant or clearly pathogenic tomorrow. ${ }^{2}$

Although at present this issue still mainly plays a role in the context of (clinical) research, it will not take long before it becomes a general issue in regular therapy and diagnostics. The integration of genomics into routine clinical practice will have a huge impact on the standard of care, including the extent to which physicians and other professionals involved can be held accountable for not communicating important new information to current or former patients. ${ }^{3}$ Basically,

physicians face the potential opportunity and responsibility, both professional and legal, for ongoing monitoring and updating of genetic-based medical advice that will be subject to constant modification throughout the patient's lifetime. ${ }^{4}$

At the same time, they have to come to terms with this responsibility, and decide what can and what cannot be expected from them, taking account of existing technological, organisational and financial constraints.

This article focuses on the legal aspects of a duty to recontact patients in the light of new (genetic) data, generated by new technologies such as NGS. In exploring this issue, we will also pay attention to different contexts (research versus care; patient versus relative), in which the duty to recontact could be at stake.

In this article, we first provide a general background on the topic of recontacting in present-day genetics, i.e., describe the circumstances in which the issue of recontacting may arise, how caregivers and patients generally view such a duty, and what can be said about current practice and the existing guidance on this point (Section 2). Subsequently, Section 3 will deal with the central question of this article, i.e., whether a possible basis for a duty to recontact could be found in the law? To what extent does such a legal duty already exist, either in general, or in specific situations? And would the answer be different for research subjects or relatives of the patient? In addressing these questions, we will not discuss the law of specific European jurisdictions, but take

2 Y.A. Stevens et al., 'Physicians' duty to recontact and update genetic advice', Personalized Medicine 14 (2017) 367-374.

3 F. Sirchia et al., 'Recontacting or not recontacting? A survey of current practices in clinical genetic centres in Europe', Eur J Hum Genet 26(7) (2018) 946-954; M. Letendre and B. Godard, 'Expanding the physician's duty of care: A duty to recontact?', Medicine and Law 23(3) (2004) 531-539.

4 Stevens et al., supra note 2, p. 369 . 
universal human rights law and principles of medical law as a starting point. Section 4 then reflects on how the duty to recontact might develop in the future, and what might be considered best practices for the current, transitory situation. Section 5 summarises the conclusions.

\section{Recontacting in Current Practice of Clinical Genetics}

\subsection{Situations in which the Need for Recontacting May Arise}

In genetics, recontacting may arise in a range of circumstances. The following situations are most often mentioned in the literature: 5

1. there is a new treatment possibility or screening recommendation that may provide new options in terms of prevention or treatment for the patient;

2. a new technique or new genetic test has become available that opens the door to a more accurate diagnosis;

3. a gene has been identified that may be relevant in relation to the disease of the patient and, the test results need to be re-evaluated by the laboratory;

4. there is a change in the guidelines leading to reclassification of test results, for instance from benign to pathogenic; the same may apply to so called 'variants of unknown or uncertain significance' (vUs), i.e. genetic variants that are of potential clinical significance, but their meaning remains as yet unclear. Obviously, the category of vus is heterogenic in the sense that they may vary from completely unknown variants to variants that are likely to be clinically relevant but where there is not yet sufficient evidence to classify them as pathogenic, with a grey area between them.

\subsection{Views of Professionals and Patients}

While recontacting could, potentially, advance important (health) interest of patients, healthcare professionals remain ambivalent. In 1999, Fitzpatrick and others found - on the basis of a questionnaire sent to 1000 geneticists in the United States and Canada - that most respondents considered recontacting patients an ethically desirable, but unfeasible goal. ${ }^{6}$ This finding is echoed

5 See E. Otten et al., 'Is there a duty to recontact in the light of new genetic technologies? A systematic review of the literature', Gen Med 17 (2015) 668-678 (671).

6 J.L. Fitzpatrick et al., 'The duty to recontact: attitudes of genetic service providers', Am J Hum Genet 64 (1999) 852-860. 
in other studies, including a survey conducted 18 years later by Carrieri and others in the United Kingdom, exploring the views of healthcare professionals and clinical scientists on recontacting former patients with new genetic information relevant to their health. While viewing recontacting as desirable under certain circumstances, most respondents expressed concerns about its feasibility. The main barriers identified were insufficient resources (lack of time, staff and financial means, as well as a lack of a suitable IT infrastructure). Another reason to be cautious with adopting a duty to recontact, is that there is a lack of clarity on which health professionals (genetic specialists, referring specialists, general practitioners) should be responsible for renewing recontact with patients in certain circumstances, and about the content and boundaries of their responsibilities. ${ }^{7} \mathrm{~A}$ third problem experienced by health professionals is that recontacting could imply the communication of unsolicited information to patients and, potentially, their relatives. The message that a previously neutral variant is pathogenic, for instance, is a potential intrusion of their privacy and may encroach upon their right not to know, even if the information is actionable in the sense that one could consider prevention or seek treatment. At the same time, if relatives would be recontacted for their benefit, this could jeopardise the patient's right to confidentiality.

With his colleagues, Carrieri also investigated the views and expectations of patients. They found that most patients viewed recontacting as desirable. This is in accordance with older literature on the information needs of patients in general: a large majority of patients want to be informed, even about smaller risks. ${ }^{8}$ This also seems to hold true for recontacting in genetics. ${ }^{9}$ However, according to Carrieri and others, there were different opinions about what type of new information should trigger recontacting. Awareness of the potential psychological impact led some to suggest that recontacting should be tailored to the nature of the information and the specific situation of patients and their families..$^{10}$ Another recent study on patient preferences to receive genetic information suggests that to the extent that patients are more informed about

7 D. Carrieri et al., 'Recontacting in clinical practice: An investigation of the views of healthcare professionals and clinical scientists in the United Kingdom', Eur J Hum Genet 25 (2017) 275-279. Since the reclassification of variants by laboratories has become an important issue, also their professional obligations and legal duties have been subject of debate. E.g. H.J. Sutherland et al., 'Cancer patients: Their desire for information and participation in treatment decisions', J Royal Soc Med 82 (1989) 260-263; E.H. Partridge et al., 'Do patients participating in clinical trials want to know study results?', J Nat Cancer Institute 95 (2003) 491-492.

$9 \quad$ See the studies mentioned by Otten et al., supra note 5, p. 676.

10 D. Carrieri et al., 'Recontacting in clinical practice: The views and expectations of patients in the United Kingdom', Eur J Hum Genet 25 (2017) 1106-1112. 
the limitations and implications of receiving unsolicited test outcomes, they become more selective in what they want to know: after receiving comprehensive background information on next generation sequencing, most participants preferred to receive only subsets of genetic information..$^{11}$ This is in line with a systematic search of the literature on stakeholder views (both professionals and patients) on communicating secondary findings in WGS and WES. This study revealed that while stakeholders were broadly supportive of returning actionable findings, experience with genetic illness and testing resulted in greater caution about receiving secondary findings. ${ }^{12}$ This finding suggests that truly informed decisions about obtaining (additional or new) information require an understanding of the implications and limitations of obtaining such information.

\subsection{Present Practice}

In light of the foregoing, it will be no surprise that recontacting is still far from being a clearly defined and widely accepted task by professionals in clinical practice. This is confirmed by studies of current practice; their message is usually that variation prevails. Although many laboratories and clinicians consider it desirable to share new relevant information with (former) patients, at least in certain circumstances, they find it difficult to elaborate clear policies and to implement obligations on recontacting in the broader context of regular care. In their study on clinical practice in the UK, Carrieri and others conclude that there are almost no policies or guidelines on this issue. Although the majority of clinical genetics services reported that they recontact patients and their family members, this is generally done in an ad hoc manner. More than half of the services were unsure whether formalised recontacting systems should be implemented. Furthermore, the majority of services do not routinely ask patients about their recontacting preferences as part of the procedure for obtaining informed consent for genetic testing. ${ }^{13}$

In 2018 Sirchia and others published the results of a web-based survey among genetic services in Europe. Their main purpose was to collect information about existing infrastructures and practices on recontacting patients.

11 R.M. Bijlsma et al., 'Cancer patients' intentions towards receiving unsolicited genetic information obtained using next generation sequencing', Familial Cancer 17(2) (2017) 309-316.

12 M.P. Mackley et al., 'Stakeholder views on secondary findings in whole-genome and whole-exome sequencing: A systematic review of quantitative and qualitative studies', Gen Medicine 19(3) (2017) 283-293.

13 D. Carrieri et al., 'Recontact in clinical practice: A survey of clinical genetics services in the United Kingdom', Gen Medicine 18 (2016) 876-881. 
From the response of 105 genetic centres (in 27 countries) they concluded that there are no standardised practices and systems in place, although the majority of the centres made clear they had recontacted patients to update them about new significant information. ${ }^{14}$ A number of barriers to the implementation of recontacting systems in case of new genetic information were mentioned, including a lack of resources and infrastructure, concerns about the potential negative psychological consequences of recontacting, unclear definitions of recontacting, ${ }^{15}$ policies that prevent health professionals from recontacting, and difficulties in locating patients after their last contact with the centre.

\section{$2.4 \quad$ Current Guidelines}

Although the number of guidelines that address recontacting is limited, mention should be made of the guidelines of the American College of Medical Genetics (ACMG) and the European Society of Human Genetics (ESHG).

In 1999 the ACMG issued a policy statement on the duty to recontact that placed the emphasis on patients' and primary care physicians' responsibility to recontact clinical genetics departments, rather than the geneticists or laboratory. ${ }^{16}$ Subsequent updates to guidelines by the ACMG have considered variant reanalysis in whole exome or whole genome screening. The most recent guidelines for the interpretation of sequence variants (2015) encourage laboratories to 'consider proactive amendment of cases when a variant reported with a near-definitive classification (pathogenic or benign) must be reclassified. ${ }^{17}$ By contrast, for vUs in the primary indication

and in the absence of updates that may be proactively provided by the laboratory, it is recommended that laboratories suggest periodic inquiry by health-care providers to determine whether knowledge of any variants of uncertain significance (.....) has changed. ${ }^{18}$

This provides a distinction between lab responsibility to re-analyse and recontact the responsible physician in the case of re-categorisation of variants, and

\footnotetext{
14 Sirchia et al., supra note 3.

15 The researchers found that there is a 'multiplicity of understandings of the term "recontacting", which respondents conflated with routine follow-up programmes, or even with post-test counselling'. Ibid.

16 ACMG, 'Duty to Re-Contact', Gen Med 1(4) (1999) 171-172.

17 S. Richards et al., 'Standards and Guidelines for the Interpretation of Sequence Variants: A Joint Consensus Recommendation of the American College of Medical Genetics and Genomics and the Association for Molecular Pathology', Gen Med 17(5) (2015) 405-424. 
clinical/patient responsibility to recontact the lab for updated information on vus.

The ESHG 'Guidelines for Diagnostic Next-Generation Sequencing' (2015) similarly imply that the lab should take the initiative in the case of reclassification of a variant. ${ }^{19}$ However, the ESHG states that 'the laboratory is not expected to re-analyse old data systematically and report novel findings, not even when the core disease gene panel changes'. On the other hand, if it is decided to change a variant from one class to another 'the lab is responsible for reanalysing the available data, re-issuing a report on the basis of the novel evidence, and also recontacting referring physicians for the patients that are possibly affected by the new status of the variant'. And: 'A system effectively linking patients and variants, and allowing for the retrieval of affected case when variants are reclassified is necessary in such a situation. ${ }^{20}$

Both the ACMG and the ESHG refer to the need for databases and mechanisms to enable laboratories to update changes and identify affected patients, as well as to the resources needed to sustain those updates.

Recontacting in Genetics: A Legal Duty?

3.1 A General Legal Duty to Recontact?

What basis might be found in the law for a duty to recontact and to what extent does such a duty already exist under present law? There is, to the best of our knowledge, no legislation in any jurisdiction that deals specifically with recontacting in clinical genetics. ${ }^{21}$ This also applies to Europe. ${ }^{22}$ However, support for the claim of a patient that he or she should have been recontacted can be found in European human rights instruments.

19 G. Matthijs et al., 'Guidelines for Diagnostic Next-Generation Sequencing', Eur J Med Genet 24 (2016) 2-5.

$20 \quad$ Ibid.

21 The most recent overview of the landscape of national legislation and policies on the return of genetic testing results (see B.M Knoppers et al., 'Return of genetic testing results in the era of whole genome sequencing', Nature Genetics 16 (2015) 553-559) does not specifically address this issue and makes no mention of legislation on this particular topic.

22 E. Rantanen et al., 'Regulations and practices of genetic counselling in 38 European countries: The perspectives of national representatives', Eur J Hum Gen 16 (2008) 1208-1216 mention recontacting as one of the topics that were 'seldom' covered in national legislation and guidance, or regarded as guided by a generally applied practice. They do not provide evidence of statutory regulation of the issue in any of the jurisdictions covered. 


\subsubsection{European Level}

The basis of the duty to recontact is to be found in the patient's basic right to receive all the health information that is available about him. That right follows first of all from the European Convention on Human Rights (1950) that provides for a right to private life (Article 8) including (as interpreted by the European Court) a right to self-determination. Exercising that right presupposes a right to learn about information relevant to one's health and wellbeing. For the area of medicine and biosciences, more specific provisions can be found in the Council of Europe's Biomedicine Convention (1997). ${ }^{23}$ Mention should be made in particular of Article 10 that states that everyone is entitled to know any information collected about his or her health. ${ }^{24}$ According to the Explanatory Report, it is crucial that individuals have access to all data that is collected about their health. ${ }^{25}$ However, it is not further specified who, the professional or the patient, should take the initiative to actually obtain these data. It should also be noted that Article 10 relates to information that has been 'collected' and is therefore more or less directly available. What this means for potential information that may be there, but needs to be obtained by re-evaluation or re-analysis of already available genetic data or genetic material, is an issue that requires further reflection.

The right to know is also incorporated in the Protocol on genetic testing for health purposes (based on the Biomedicine Convention) which states in Article 16 that the person who asks for genetic testing is entitled to know any information collected about his or her health derived from a test. ${ }^{26}$ That right can under specific circumstances be subject to certain restrictions, but only in the interest of the person concerned, according to the Protocol's Explanatory Report.

With regard to the Biomedicine Convention itself, reference should also be made to Article 4 that stipulates that all health professionals have to take good care of their patients and act in accordance with professional responsibilities.

23 Not all Council of Europe member states signed the Biomedicine Convention, such as the United Kingdom and Belgium. Other countries, such as the Netherlands, signed the convention, but did not ratify it.

24 See also C. Ploem, 'Handling unsolicited findings in clinical care: A legal perspective', Eur J Health Law 21 (2014) 489-504 (493-494).

25 The right to have access to one's personal data is also a basic element of privacy law, and incorporated in the General Data Protection Regulation (GDPR) of the European Union that entered into force in May 2018.

26 The Protocol contains only general ethical and legal principles concerning genetic testing and therefore makes no distinction between the different types of findings, such as with clear implications for patients (and/or their relatives) and findings of which the relevance is unknown or unclear. 
The Explanatory Report explains that, although the same medical duties may vary slightly from one society to another, the fundamental principles of the practice of medicine apply universally. All professionals in the field of medicine must act with care and competence, and pay careful attention to the needs of each patient.

\subsubsection{National Level}

This last point - that the care given (including the information to be provided) should be tailored to individual needs - runs parallel with developments at national level, where legislation and/or court decisions in several jurisdictions acknowledge that in patient care an individualised approach is appropriate and that the needs of the individual patient should be guide what information is provided to them. ${ }^{27}$

It is broadly accepted that physicians are also responsible to provide continuity of care, including adequate post-treatment care. In the law, it is generally acknowledged that in a relationship between two parties (contractual or otherwise), parties have to be aware of their mutual interests, unless what is expected is unreasonable or when doing justice to those interests requires a disproportional effort. ${ }^{28}$ This requirement - to consider and eventually to act upon the justified expectations of the other party (in this case the patient) may, in some circumstances, also result in obligations after the relationship has ended. At the same time, it is obvious that if there is no longer an ongoing relationship between patient and caregiver, this does have an impact on the extent and nature of the duty of good care. This underlines the importance of clarity towards the patient about what to expect after discharge.

Is the foregoing sufficient for the acceptance of a duty to recontact? It demonstrates that receiving information relevant to one's health is regarded as a fundamental interest of each individual, sick or healthy; that this interest is protected by the law and that on the basis of the law, healthcare professionals have certain responsibilities towards their (former) patients. One could also reason that patients might always expect that their care givers do what they reasonably can to meet their informational needs. However, it is still unclear what may be reasonably expected from health professionals if the information concerned is not readily available and recontacting would for instance require

27 See e.g. C. Mitchell et al., 'Exploring the potential duty of care in clinical genomics', Med Law International 17(3) (2017) 158-182.

28 The answer to the question what can be reasonably expected from a healthcare professional also has an ethical dimension; see for that perspective for instance the articles on the disclosure of genetic results to research participants in a special issue of the American Journal of Bioethics: Am J Bioethics 6(6) (2006). 
considerable efforts in terms of re-evaluating and re-analysing available data and material, in some cases not only once but repeatedly over a long period of time. Therefore, apart from other concerns (such as difficulties in contacting former patients and possible interference with their right not to know), given the effort burden of updating previous test results, it cannot be argued that patients have an unconditional right to be recontacted under all circumstances. It is therefore not surprising that a general responsibility to recontact patients has not, so far, been incorporated in legislation, articulated by the courts, or embodied in professional standards. ${ }^{29}$

This is in line with the position most often adopted in the international literature; accepting a general duty to recontact could impose an onerous and inequitable burden on health professionals, with significant malpractice implications. A similar position is taken by Letendre and Godard:

The recognition of a duty to recontact in the context of medical genetics would, at this point, put health professionals at an increased risk of medical negligence since they do not possess the resources or the knowledge to assess and fulfil such a duty. ${ }^{30}$

\subsection{A Duty to Recontact in Specific Circumstances?}

The question remains whether there are no specific circumstances in which a legal duty to recontact would clearly exist. First of all, the acknowledgment of a responsibility to recontact by the profession is not necessary for establishing a legal duty. As Rothstein rightly points out: 'Whether the law recognises a duty to notify patients probably will be based on the relative burdens and benefits, rather than whether the medical profession recognises the duty'. ${ }^{31}$ Furthermore, the fact that so far no court in Europe (or North America ${ }^{32}$ ) has accepted - as far as we know - a duty to recontact in clinical genetics, does not necessarily mean that a court may not identify such a duty in a particular case and apply that duty to the situation under review. 'Health care providers

29 See A.G.W. Hunter et al., 'Ethical, legal and practical concerns about recontacting patients to inform them of new information: The case in medical genetics', Am J Med Gen 103(4) (2001) 265-276 (269-270).

$30 \quad$ Letendre and Godard, supra note 3, p. 537.

31 M.A. Rothstein, 'Physicians' duty to inform patients of new medical discoveries: The effect of health information technologies', J Law Med Ethics 39(4) (2011) 69o-693 (692).

32 A first, interesting case maybe a 2016 lawsuit in the US (Williams v Athena), pending in a South Carolina federal court, involving allegations of negligent variant interpretation by a genetic testing laboratory; see A. Thorogood et al., 'Public variant databases: Liability?' Gen Med 19 (2017) 838-841 and 'Legal update: Lawsuit raises questions about variant interpretation and communication', Am J Med Gen A. 173(4) (2017) 838-839. 
therefore cannot be content relying only on existing established legal duties, but must also look proactively ahead for new duties courts may impose', as Stevens and others comment. ${ }^{33}$

In which circumstances might a court decide that in retrospect the caregiver (or the laboratory that did not inform the latter) owed a duty to inform the patient? Apart from the existence of a guideline applicable in the specific situation, or, a case where the laboratory or healthcare provider might have themselves raised the expectations of patients, the most plausible answer (which would seem to apply in most, if not all jurisdictions) is that this is most likely to occur when recontacting would be of significant clinical relevance for the patient. In such a situation, unless the efforts and costs involved would be disproportional, a judge could come very well to the conclusion that a caregiver owes a duty to inform his patient. This means in more concrete terms that if there is a finding which is analytically valid and of clear and substantial clinical utility, it should somehow be communicated to the patient, unless this would be too complicated or burdensome. In the context of the new genetics, the latter may in particular be the case when periodic re-evaluation or re-analysis of genetic data or material would be needed. Furthermore, the time elapsed since initial testing is likely to have a certain bearing on this: the more time has passed, the less likely it would seem that a professional duty will be found.

Basically, what is at stake here is not so much the general duty to recontact, but a more limited one, i.e. to warn patients (in principle also former patients) for predictable and preventable harm, a duty that has been recognised in several other situations. In this sense, a specific duty to recontact is not completely new. The situation in which such a duty to recontact would arise is also very similar to the one in which a healthcare provider should inform patients about incidental, unsolicited findings. In the latter case, according to an extensive literature, a duty to inform can be assumed if reasonable possibilities in the clinical or preventive domain are available and the disclosure of the findings could prevent harm to the patient's health or well-being. ${ }^{34}$ Of course the two situations are not completely the same: recontacting relates to later findings, most often concerning patients already discharged from care; furthermore, it may require considerable efforts in that it involves the updating of previous test results. On the other hand, those findings will more often be related to the original request for care than unsolicited findings will. Both situations are about new or extra information, however, and in both cases the professionals involved may not remain silent if the information is of crucial importance to

33 Stevens et al., supra note 2, p. 371.

34 Ploem, supra note 24. 
the health of identifiable patients and the efforts to inform them are not disproportional. One could also argue: the more significant the finding, the more effort professionals should put in trying to contact former patients.

\subsection{A Legal Duty to Recontact Participants in Research and Relatives?}

Another issue is whether the above holds only for (current and former) patients, or also for participants in research and relatives of patients. May they also expect to be warned of new genetic information relevant to their health, and if so, by whom? By their initially diagnosed relative or - directly - by his or her treating physician? ${ }^{35}$ An issue in this particular context that we do not discuss here is that it will be often unknown to the professional who is potentially responsible for recontacting a research participant or relative, what information they would, or would not, prefer to receive. The latter should be a matter of concern to professionals because each individual has an interest, protected by the law, not to know certain information about himself. ${ }^{36}$

With regard to research subjects, two situations can be distinguished: one in which care and research go hand in hand (and the patient is also a subject of research), and the other in which the research subject is not a patient, but a healthy volunteer. In the first situation, the research subject, because he is also a patient, is entitled to the same approach as any other patient. When researchers adopt roles traditionally held by clinicians, they must also accept the duty to recontact study participants when new information is discovered (at least within the limits set out above). ${ }^{37}$ This way of approaching research subjects follows also from the Declaration of Helsinki which holds that when physicians combine medical research with medical care, this may not adversely affect the health of the patients who serve as research subjects. ${ }^{38}$

As to the second situation, it is important to keep in mind that research is distinct from care. A researcher does not owe the same duties to a research participant as a caregiver to a patient. But do researchers have responsibilities when the reporting of individual research results is concerned? In the literature on the disclosure of individual findings in genetic research, there is no consensus on the scope of researchers' duties vis-à-vis participants. ${ }^{39}$ However, given

35 See M.A. Rothstein, 'Reconsidering the duty to warn genetically at-risk relatives', Genet Med 20(3) (2018) 285-290.

36 C. Ploem, 'The Right Not to Know: Scope and Limits', Med Law 36(2) (2017) 81-9o.

37 C.H. Wade et al., 'When do genetic researchers have a duty to recontact study participants?', AmJ Bioethics 6(6) (2006) 26-27.

38 See principle 14 Declaration of Helsinki of the Wold Medical Association, Fortaleza, Brazil October 2013 .

39 See in particular the special issue of the AmJ Bioethics 6(6) (2006) on this topic. 
the different position of researchers as compared to care givers and taking account of the problems associated with recontacting in genetics, it is unlikely that, at this point in time, courts would find a duty to recontact research subjects. If at all, this could only occur in exceptional situations in which a direct threat of serious harm to a person's health is discovered that could relatively easy be avoided by warning the person concerned. ${ }^{40}$

With regard to the relatives of patients, a qualified answer to the question whether or not they should be informed, seems justified. As long as patients are alive, the guideline in most countries is that they, or their surrogates (family members, legal guardian) may expect to be informed. In which case, it is first of all the responsibility of the patient or his representative(s) to inform the relatives who might be carriers of (a) pathogenic gene(s). ${ }^{41}$

After the death of a patient, certain information might still be of crucial importance to relatives. In that case, it is hard to see why the relatives in question should not be entitled to the same information, provided that it has become available not too long after the patient has deceased and that the family can be traced without much difficulty. ${ }^{42}$

\section{Future Developments and How to Proceed in the Meantime}

Is the duty to recontact likely to expand in the future? According to Stevens and others, it is likely that genomic information and DNA-sequence data will become a standard component of clinical practice within the next decade and that physicians will be held responsible in more circumstances for updating clinical advice in response to evolving genetic knowledge. ${ }^{43}$ Together with these authors, we believe that the duty to recontact may gradually expand in the future.

There are three factors that are likely to have a bearing on this process:

1 developments in medical genetics: the increasing relevance of genetic information in conventional care/mainstreaming of genetics,

$40 \quad$ Ploem, supra note 24, p. 504.

41 See for instance Article 18 of the Additional Protocol to the Biomedicine Convention concerning Genetic Testing for Health Purposes, 2008 ('Where the results of a genetic test undertaken on a person can be relevant to the health of other family members, the person tested shall be informed'.). See also H.D.C. Roscam Abbing, 'Genetic Testing for Health Care Purposes, a Council of Europe Protocol', Eur J Health Law 15 (2008) 353-359.

42 This situation may arise in case of lung cancer, for instance, where life expectancy is short.

Stevens et al., supra note 2, p. 372. 
the general application of WES/WGS in clinical care, the growing extent to which testing is viewed as on ongoing process rather than as a one-off resource;

the changing landscape of healthcare in general: the advance of personalised medicine, together with a changing doctor-patient relationship in which the physician helps the patient in identifying and monitoring individual health risks and offers advice on lifestyle and prevention;

3 developments in the IT infrastructure, including the introduction of personal electronic health records, which will facilitate the review of already available data, in particular when variants can be updated automatically, and the communication between professionals and with patients, at the same time allowing for more active participation of patients in the management of their own health, for instance by receiving updates of previous test results.

This last factor especially may have consequences for the establishment of legal duties, because health information technology has the potential to reduce the burden of patient notification, thereby changing the balance of the burdens and benefits in recontacting. ${ }^{44}$ At the same time, it may also enable patients to play their role. In the view of many commentators, the responsibility to communicate new genetic information should be a shared one in which patients have to participate, along with general practitioners, medical specialists and laboratories. ${ }^{45}$

The further development of a legal duty to recontact will of course also be influenced to a large extent by the (further) articulation of the professional standard in this field. Courts will always look at how the profession itself has struck the balance between the desirability and the feasibility of recontact and what it considers best practice. But the elaboration of professional guidelines does not always protect the profession from malpractice claims or other law suits. In a way, guidelines may make the profession more vulnerable to the extent that specific responsibilities are acknowledged. On the other hand, it is the most effective way to manage expectations and to explain what will, and what will not, be provided by the profession. To cite Rothstein again: 'Avoiding notification responsibilities will not necessarily forestall potential legal liability;

44 Rothstein, supra note 35, p. 691.

45 J. Clarke et al., 'Look back: The duty to update genetic counselling', in: B.M Knoppers et al. (eds.), Human DNA: Law and Policy; International and Comparative Perspectives (The Hague: Kluwer Law Internationals, 1997), pp. 121-132; see also Letendre and Godard, supra note 3 . 
assuming reasonable duties probably will decrease the risk of liability' ${ }^{46}$ In other words: although caution is warranted in the recognition of responsibilities, one should not refrain from doing so.

If the current situation is one of transition, the question is then what to do in the present situation in which there is as yet so much uncertainty about needs, preferences, practical possibilities, roles and the related responsibilities.

In the literature, this point is addressed by several authors included in the review carried out by Otten and others in 2014 and $2015 .{ }^{47}$ But mention should be made in particular of the more recent publications of Carrieri and others, based on extensive and repeated studies on the situation in the UK. ${ }^{48}$ They recommend as a first step that recontacting is routinely discussed with patients and that they should be informed that they are welcome to contact the team if a potentially relevant event occurs (e.g. a child reaching reproductive age) or, if agreed, at regular intervals based on the specific condition. This renewed contact initiated by the patient may also trigger a review of the patient's file by clinicians to check it on new information that is relevant to him or her. ${ }^{49}$ Giving patients an important role in the process of recontacting allows them more control over that process and makes it easier for professionals to meet their expectations.

Patients who participated in a study carried out by Dheensa and others expressed similar views. They suggested a 'joint venture model' in which efforts to recontact are shared with healthcare professionals. Like Carrieri and others, the authors of this study advise that - as a first step to delineating responsibilities in the clinical setting - professionals address the issue of recontacting with their patients, including which new information should trigger the professional to initiate recontact, as part of the consent process for genetic testing. ${ }^{50}$

Discussing this issue with patients is certainly a step that can and should be taken in the present situation. However, it should be combined with a gradual, further development of professional guidelines, also at the level of (groups of) diagnosis. This not only with a view to the management of the expectations of patients, but also to further delineate the respective roles of all parties involved (professionals and patients) and to allocate responsibilities.

\footnotetext{
$46 \quad$ Rothstein, supra note 35, p. 692.

47 Otten et al., supra note 5, 2015.

48 Carrieri et al., supra notes 7 and 10.

49 D. Carrieri et al., 'Recontacting in clinical genetics and genomic medicine? We need to talk about it', European Journal of Human Genetics 25 (2017) 520-521.

50 S. Dheensa et al., "A "joint venture" model of recontacting in clinical genomics: Challenges for responsible implementation', Eur J Med Genetics 60 (2017) 403-409.
} 


\section{5}

\section{Conclusion}

Returning to the main question of this article: is a (legal) duty to recontact a patient when new genetic information becomes available 'futuristic'? On the basis of our research, the general answer is affirmative, at least with regard to an unconditional duty of that kind in the context of genetics. In many cases, apart from other concerns, recontacting would require re-evaluation or re-analysis of the available data. Taking the existing technological, organisational and financial constraints associated with updating previous test results into account, it is hard to see how, at least at this point in time, there could be a general duty that would be legally enforceable. This is also the consensus in the international literature on the topic. And so far, there are no jurisdictions in which such a duty has been accepted, either by the legislator or by the courts.

On the other hand, health professionals should always do what can reasonably expected from them to do justice to the patient's rights to information and good care. When there is reason to believe that recontacting would be of significant clinical relevance for the patient, they should do so, unless the efforts and costs involved would be disproportional. In such a situation, a judge could come very well to the conclusion that a caregiver owes a duty to inform his patient. It could be argued that a comparable duty to warn is also owed to the relatives of (recently) deceased patients, or to patients participating in some forms of research.

As NGS/WES and the processing of DNA-sequence data become a more standard component of clinical practice - a development that is currently taking place in cancer diagnostics and treatment -, it is to be increasingly expected that situations will arise in which physicians are held responsible for updating clinical advice in response to evolving genetic knowledge. In light of this, the duty to recontact is likely to gradually expand and may even transform into a more general legal duty, essentially reflecting the norm that the more significant the finding, the more effort may be expected of professionals. Developments in health information technology are likely to have a bearing on this process, to the extent that they will reduce the burden of updating previous test results and communicating new information, including to former patients. 